\title{
THE DYNAMIC MODEL ENSURING EFFECTIVE CONTRACTS FOR ACADEMIC AND TEACHING STAFF ${ }^{1}$
}

\author{
Nadezhda A. Kizatova \\ Volgograd State University, Volgograd, Russian Federation \\ Nataliya V. Loseva \\ Volzhsky Institute of Humanities - Branch of Volgograd State University, \\ Volzhsky, Russian Federation \\ Vasiliy V. Tarakanov \\ Volgograd State University, Volgograd, Russian Federation
}

\begin{abstract}
The results of the research presented in this article are within the framework of the research area engaged in development of basic provisions and methodologies for managing educational institutions and, in particular, working out methods and algorithms for financial analysis and educational and research management in universities. Drawing upon the per-capita normative financing methodology, which is currently used in Russia, as well as the structure of the existing and prospective educational standards and curricular, main provisions of strategic development plans for university structural subdivisions, the authors propose a dynamic financial and mathematical model of building salary funds for departments, institutes and university in general. The article specifically describes the methodology of financial support of effective medium-term contracts for academic and teaching staff. The practical implementation of this model will result in higher motivation of the staff and will ensure improvement in educational institutions performance. An effective contract contributes to better staff's performance, their input in implementing strategies, programmes and projects for developing institutes, departments and university as a whole, which gives rise to the effectiveness of the whole educational institution. Besides, the developed dynamic financial and mathematical model makes it possible to offer methodology for planning financial support for implementing effective medium-term contract for academic and teaching staff. The methodology takes into account the specific features of educational process organization in the long-term, the incorporation of various disciplines in the curriculum, with possible curriculum changes during the planning period, the student contingent adjustment, and admissible integration of different student groups into academic shifts. In its turn, all this provides an educational $=$ institution with an opportunity of planning a long-term educational process with optimal parameters.

¿ Key words: effective contract, financial support, algorithms of managing financial activities of a higher educational institution, per-capita normative financing, a model of building a salary fund.

ДИНАМИЧЕСКАЯ МОДЕЛЬ ОБЕСПЕЧЕНИЯ ЭФФЕКТИВНЫХ КОНТРАКТОВ НАУЧНО-ПЕДАГОГИЧЕСКИХ РАБОТНИКОВ ${ }^{1}$
\end{abstract}

\section{Надежда Александровна Кизатова}

Волгоградский государственный университет, г. Волгоград, Российская Федерация

\section{Наталия Владимировна Лосева}

Волжский гуманитарный институт (филиал) Волгоградского государственного университета, г. Волжский, Российская Федерация 


\title{
Василий Валерьевич Тараканов
}

Волгоградский государственный университет, г. Волгоград, Российская Федерация

\begin{abstract}
Аннотация. Результаты исследований, изложенные в настоящей статье, лежат в рамках научного направления, занимающегося разработкой основных положений и методик менеджмента образовательных организаций, в частности созданием методов и алгоритмов финансового анализа и управления образовательным и научно-исследовательскими процессами в университетах. На основе действующей в России методики нормативно-подушевого финансирования образовательных учреждений, структуры существующих и разрабатываемых образовательных стандартов, учебных планов образовательных программ, основных положений стратегических планов развития структурных подразделений вузов авторами предложена динамическая финансово-математическая модель формирования фондов оплаты труда кафедр, институтов (факультетов) и вуза в целом. В частности, в работе описана методика финансового обеспечения эффективных контрактов научно-педагогических работников на среднесрочный период. Практическая реализация данной модели позволит повысить мотивацию научно-педагогических работников вуза и обеспечит планирование мероприятий по улучшению различных показателей деятельности учебного заведения. Эффективный контракт способствует увеличению результативности труда работников, их вклада в реализацию стратегий, программ и проектов развития институтов (факультетов), всего вуза, что влечет за собой рост эффективности и всей образовательной организации. Кроме того, построенная динамическая финансово-математическая модель позволяет предложить методику планирования финансового обеспечения процесса реализации эффективных контрактов научно-педагогических работников образовательного учреждения на среднесрочный период. Методика учитывает особенности организации учебного процесса в перспективе, насыщенность учебного плана различными дисциплинами с учетом возможности изменения учебного плана в периоде планирования, корректировку контингента обучающихся, допустимость объединения в потоки различных групп студентов, а это, в свою очередь, дает возможность планировать в образовательной организации учебный процесс с оптимальными параметрами на длительный период.
\end{abstract}

Ключевые слова: эффективный контракт, финансовое обеспечение, алгоритмы управления финансовой деятельностью вуза, нормативно-подушевое финансирование, модель формирования фонда оплаты труда.

Реформирование системы высшего образования в Российской Федерации имеет своей целью повышение качества образовательных услуг и эффективности использования финансовых, материально-технических, организационных ресурсов. Это влечет за собой изменения методик экономического анализа и алгоритмов финансового управления образовательной организацией в целом. Первоочередными становятся задачи эффективной организации образовательного процесса и методы оптимального финансового управления вузами. Назрела необходимость разработки соответствующих методик, алгоритмов и технологий системы менеджмента вуза, которые позволили бы образовательной организации в новых экономических и социальных условиях в полной мере использовать свой потенциал. В частности, в работах $[2 ; 3 ; 11]$ были предложены финансово-математические модели бюджетирования структурных подразделений, включающие новый алгоритм формирования фонда оплаты труда профессорско-преподавательского состава (далее ФОТ ППС) кафедр вуза. Но эти исследова- ния не касались вопросов динамики контингента студентов и изменения структуры образовательных программ вуза. Динамическая модель формирования ФОТ ППС кафедр вуза была построена в [1]. Впрочем, нерассмотренной осталась проблема, связанная с обеспечением эффективных контрактов.

Известно, что определяющим документом, описывающим особенности структуры и содержания эффективного контракта, является «Программа поэтапного совершенствования системы оплаты труда в государственных (муниципальных) учреждениях на 20122018 гг.», утвержденная распоряжением Правительства РФ от 26 ноября 2012 г. № 2190-р [6]. В ней сказано, что «эффективный контракт - это трудовой договор с работником, в котором конкретизированы трудовые обязанности, условия оплаты труда, показатели и критерии оценки эффективности деятельности для назначения стимулирующих выплат в зависимости от результатов труда и качества оказываемых государственных (муниципальных) услуг, а также меры социальной поддержки». 
Вопросам практики внедрения эффективного контракта в деятельность образовательных организаций в последние годы посвящено много исследований. Однако в них, как, например, и в работах [4; 5-9], изучались в основном проблемы реализации эффективного контракта в сфере трудовых отношений и механизмы их решения в вузах, роль эффективного контракта в маркетинговой политике вуза.

Вопросы финансового обеспечения эффективных контрактов научно-педагогических работников рассмотрены в [10], но в этой работе не затронуты задачи планирования работы на перспективу, что очень важно для руководства вузов, то есть расчет возможности финансирования структурных подразделений по различным направлениям, в том числе и для обеспечения эффективных контрактов, на несколько лет вперед.

Основной целью данной статьи является теоретическая разработка динамической модели финансового обеспечения эффективных контрактов, алгоритма планирования и формирования бюджета структурных подразделений вуза в части оплаты работ по эффективным контрактам в перспективе, а также формирование соответствующей методики планирования финансового обеспечения реализации эффективных контрактов научно-педагогических работников образовательных учреждений.

План работы любого высшего учебного заведения требует определения предполагаемых затрат на обеспечение всех видов деятельности. При этом исходить вуз может из базовых нормативных затрат на выполнение государственного задания, а также дополнительных источников финансирования (если таковые имеются). Но размеры составляющих базовых нормативных затрат не являются для вузов обязательными, скорее отражают статистическую ситуацию по стране. Поэтому распределение средств по видам деятельности - это задача, которую каждое учебное заведение решает самостоятельно. Следовательно, определение размеров составляющих базовых нормативных затрат, создание каких-либо методик является важнейшим элементом современной экономики российского образования.
Базовая часть нормативных затрат (в руб. за одного обучающегося) ежегодно определяется соответствующими решениями министерства образования и науки и зависит от направлений подготовки, конкретных образовательных программ (ОП). Здесь и далее под ОП вуза будем понимать программы бакалавриата, специалитета, магистратуры или аспирантуры, характеризуемые направлением подготовки, годом набора, нормативным сроком обучения (количеством курсов). Базовая часть нормативных затрат, очевидно, включает в себя нормативную часть финансирования ОП (в руб. за одного обучающегося), направляемую вузом в ФОТ ППС, нормативную часть финансирования ОП, выделяемую вузом на обеспечение эффективных контрактов, а также нормативную часть финансирования ОП, связанную с материально-техническим обеспечением. Все эти нормативные части могут отличаться как для разных ОП, так и для различных потоков одной и той же ОП.

В работе [1] была построена модель формирования ФОТ ППС кафедры для прогнозирования его величины в будущем. Беря за основу работы $[1 ; 10]$, построим финансово-математическую модель формирования частей бюджетов кафедры, института и университета, создаваемых для обеспечения эффективных контрактов, с целью прогнозирования их величины в будущем. Год построения прогноза будем считать начальным моментом времени $(t=0)$, следующий учебный год $t=1$ и так далее до желаемой глубины прогноза (не более 6 лет).

При действующем сейчас нормативноподушевом финансировании образовательных организаций ее бюджет, а следовательно, и бюджеты структурных подразделений существенно зависят от контингента обучающихся. Очевидно, что количество студентов на каждой ОП вуза зависит от многих факторов и не может быть постоянной величиной.

Обозначим за $K(i, j, t)$ - контингент студентов, обучающихся на $j$-м курсе $i$-й ОП, переменной $i$ будем присваивать значения в соответствии с классификатором. Переменная $t$ отвечает за год планирования. Тогда планируемый набор на первый курс любой ОП имеет вид $K(i, 1, t)$, для направлений подготовки 
бакалавров $K(i, 5, t)=K(i, 6, t)=0$ для направлений подготовки магистров $K(i, 3, t)=\ldots=$ $K(i, 6, t)=0$.

Аналогично определяются значения контингента для других направлений подготовки (специалитет, магистратура, аспирантура) с учетом форм обучения (очная, очно-заочная, заочная). В случае когда в начальный момент времени $(t=0)$ некоторая ОП с номером $i_{0}$ только планируется к открытию, а набор будет осуществлен лишь через 2 года, тогда при любом значении $j$

$$
K\left(i_{0}, j, 0\right)=K\left(i_{0}, j, 1\right)=0, K\left(i_{0}, 1,2\right)=v
$$

где $v$ - величина планируемого набора.

Как отмечалось выше, контингент студентов каждого курса - величина, зависящая от многих факторов, и она не может быть постоянной величиной. Поэтому следует проанализировать его динамику за предыдущие несколько лет и ввести в рассмотрение коэффициент изменения контингента $\delta(i, j, t) j$-го курса $i$-й ОП для года $t$ [1]. Тогда для курсов, начиная со 2-го, очного отделения в году планирования $t$ количество студентов определяется следующим образом:

$$
K(i, j, t)=K(i, j-1, t-1) \cdot \delta(i, j-1, t-1) .
$$

Разумеется, поскольку количество студентов должно быть натуральным числом, берем целую часть этого произведения. Заметим, что этот коэффициент может быть тесно связан с конкретным набором данной ОП, в этом случае зависимость коэффициента изменения контингента от года планирования можно получить на основе экспертных оценок успеваемости студентов конкретного потока.

Основываясь на алгоритмах, изложенных в $[1-3 ; 10 ; 11]$, определим через трудоемкости компонент учебного плана (далее - УП) долю $p$-й кафедры в обучении студентов $i$-й ООП, реализуемой в $j$-м году обучения в зависимости от года планирования $t$. Для этого обозначим $\operatorname{Tr}(i, j, k, t)$ - трудоемкость (измеряемую в зачетных единицах) $k$-й дисциплины (компонента учебного плана) $i$-й ОП, читаемой на $j$-м курсе для года планирования $t$. Поскольку учебный план может меняться, то и трудоемкость каждой дисциплины, очевидно, должна зависеть от $t$.

Используем в построении модели параметр $k o e f(i, j, k, t)$ - коэффициент трудоемкости компонента учебного плана, который учитывает специфику организации учебного процесса при преподавании данной дисциплины. Он полностью аналогичен коэффициенту, применяемому в стационарных моделях $[2 ; 10 ; 11]$ и введенному в динамической модели в [2].

Далее обозначим $N(i, j, t)$ - количество компонент учебного плана $i$-й ОП на $j$-м курсе в соответствии с годом планирования $t$. В силу того что в УП можно вносить изменения, величина $N(i, j, t)$ обязательно должна зависеть от $t$.

Таким образом, приведенная трудоемкость:

$$
\operatorname{Tr}_{p r}(i, j, t)=\sum_{k=1}^{N(i, j, t)} k o e f(i, j, k, t) \cdot \operatorname{Tr}(i, j, k, t)
$$

а доля каждого компонента УП определяется отношением:

$$
D(i, j, k, t)=\frac{\operatorname{Tr}(i, j, k, t)}{\operatorname{Tr}_{p r}(i, j, t)} .
$$

Кроме того, как и в [1], с помощью индикатора $\operatorname{Ind}(i, j, k, t, p)$ выделим из всех компонент УП те, которые обеспечивает кафедра с номером $p$. Значение индикатора лежит в промежутке [0;1] и равно доли участия кафедры $p$, если $k$-й компонент плана $i$-й ООП на $j$-м курсе обеспечивается несколькими кафедрами; $\operatorname{Ind}(i, j, k, t, p)=0$, если эта кафедра не обеспечивает данный компонент; $\operatorname{Ind}(i, j, k, t, p)=1$, если обеспечивает его полностью.

Итак, доля кафедры $p$ в УП $i$-й ООП на $j$-м курсе в зависимости от года планирования $t$ выглядит следующим образом:

$$
D_{k a f}(i, j, t, p)=\sum_{k=1}^{N(i, j, t)} D(i, j, k, t) \cdot \operatorname{Ind}(i, j, k, t, p) .
$$

Ранее отмечалось, что в базовую часть нормативных затрат входят составляющая, направляемая вузом в ФОТ ППС; составляющая, выделяемая вузом на обеспечение эффективных контрактов; нормативная часть фи- 
нансирования ОП, связанная с материальнотехническим обеспечением и др.

В [1] была получена динамическая модель определения ФОТ кафедры, если считать заданными составляющую базовых нормативных затрат, отправляемую в ФОТ ППС $\operatorname{Norm}(i, j, t)$ и $N$ - количество ОП вуза. Оставим эти предположения в силе. Фонд оплаты труда $p$-й кафедры в году планирования $t$ для обеспечения учебной деятельности составит

$$
\begin{aligned}
& \operatorname{Fot}(p, t)=\sum_{i=1}^{N} \sum_{j=1}^{6} D_{k a f}(i, j, t, p) \cdot K(i, j, t) \cdot \\
& \cdot \operatorname{Norm}(i, j, t),
\end{aligned}
$$

где $K(i, j, t)$ - значение контингента, вычисленное по формуле (1).

В [10] было предложено нормативную часть финансирования ОП (в руб. за одного обучающегося), выделяемую вузом на обеспечение эффективных контрактов, разделить на три части с учетом того, что текущие и перспективные планы развития кафедр, факультетов (институтов) и вуза могут различаться в связи с разделением видов работ и ответственности. Тогда для данного норматива будет справедлива формула:

$$
\begin{gathered}
\operatorname{Norm}_{e k}(i, j, t)=\operatorname{Norm}_{e k}(v, i, j, t)+ \\
+\operatorname{Norm}_{e k}(f, i, j, t)+\operatorname{Norm}_{e k}(p, i, j, t),
\end{gathered}
$$

где $\operatorname{Norm}_{e k}(v, i, j, t)$ - нормативная часть финансирования $i$-й ОП, реализуемой в $j$-м году обучения, для года планирования $t$ (в руб. за одного обучающегося), выделяемая вузом на обеспечение эффективных контрактов в соответствии с планами развития вуза; $\operatorname{Norm}_{e k}(f, i, j, t)$ - нормативная часть финансирования $i$-й ОП, реализуемой в $j$-м году обучения, для года планирования $t$ (в руб. за одного обучающегося), выделяемая вузом на обеспечение эффективных контрактов в соответствии с планами развития факультетов (институтов); $\operatorname{Norm}_{e k}(p, i, j, t)-$ нормативная часть финансирования $i$-й ОП, реализуемой в $j$-м году обучения, для года планирования $t$ (в руб. за одного обучающегося), выделяемая вузом на обеспечение эффективных контрактов в соответствии с планами развития кафедр.

Тогда для формирования части бюджета кафедры с целью обеспечения эффективных контрактов будем применять следующую формулу:

$$
\begin{aligned}
\text { Fot }_{e k}(p, t)= & \sum_{i=1}^{N} \sum_{j=1}^{6} D_{k a f}(i, j, t, p) \cdot K(i, j, t) \cdot \\
& \cdot \operatorname{Norm}_{e k}(p, i, j, t)
\end{aligned}
$$

где величины $D_{k a f}(i, j, t, p), K(i, j, t)$ - определены ранее и полностью зависят от учебных планов и особенностей организации учебного процесса.

Для формирования части бюджета факультета (института), предназначенной для обеспечения эффективных контрактов в соответствии с планами развития факультета $\left(F o t_{e k} F(f, t)\right)$, будем применять следующую формулу:

$$
F_{\text {ot }} F(f, t)=\sum_{i=1}^{N_{f}} \sum_{j=1}^{6} K(i, j, t) \cdot \operatorname{Norm}_{e k}(f, i, j, t),
$$

где $N_{f}$ - число ОП вуза, в реализации которых участвует факультет с меткой $f$.

Аналогично для вычисления части фонда оплаты труда, предназначенного для обеспечения эффективных контрактов в соответствии с планами развития вуза $\left(F_{o t} V(p, t)\right)$, будем применять формулу:

$$
\operatorname{Fot}_{e k} V(p, t)=\sum_{i=1}^{N} \sum_{j=1}^{6} K(i, j, t) \cdot \operatorname{Norm}_{e k}(v, i, j, t),
$$

$N$ - количество ОП вуза; $K(i, j, t)$ - значение контингента, вычисленное по формуле (1).

Заметим, что бюджеты факультетов и вуза, направляемые на обеспечение эффективных контрактов, не зависят от особенностей организации учебного процесса, но существенно зависят от числа студентов.

Первостепенной задачей проводимого исследования является создание единой дескриптивно-оптимизационной модели образовательной и финансово-хозяйственной деятельности вуза и разработка на ее основе методов и алгоритмов финансового анализа и управления образовательным процессом в университетах. В данной статье разработана динамическая финансово-математическая модель формирования фонда оплаты труда научно-педагогических работников вузов для обеспечения эффективных контрактов. В ее основе лежат существующие принципы и механизмы функционирования образовательных 
организаций, в том числе методика нормативно-подушевого финансирования деятельности вузов по реализации государственного задания. Предполагается возможность применения разрабатываемой методики бюджетирования структурных подразделений в любом университете России. Она позволяет устранить противоречия между принципами финансового обеспечения деятельности образовательной организации в целом и отдельных ее структурных подразделений в различных видах деятельности и осуществлять планирование финансовой составляющей работы вуза в перспективе.

\section{ПРИМЕЧАНИЕ}

1 Исследование выполнено при финансовой поддержке РФФИ и Администрации Волгоградской области, проект «Финансово-математические модели и алгоритмы бюджетирования структурных подразделений образовательных организаций» № 17-12-34015.

\section{СПИСОК ЛИТЕРАТУРЫ}

1. Кизатова, Н. А. Динамическая модель формирования фонда оплаты труда профессорско-преподавательского состава вузов / Н. А. Кизатова //Современная экономика: проблемы и решения. 2016. - № 7 (79). - С. 15-24.

2. Корольков, С. А. Модель оптимального планирования штата профессорско-преподавательского состава кафедр вуза / С. А. Корольков, А. Г. Лосев, И. М. Решетникова // Вестник Волгоградского государственного университета. Серия 3, Экономика. Экология. - 2013. - № 1. - С. 149-154.

3. Корольков, С. А. Управление образовательными программами через внедрение учебно-финансовых планов / С. А. Корольков, А. Г. Лосев, В. В. Тараканов // Университетское управление: практика и анализ. - 2016.- № 4 (104) . - С. 49-55.

4. Ленгардт, В. А. Эффективный контракт в маркетинговой политике университета / В. А. Ленгардт, Л. Ю. Шемятихина // Фундаментальные исследования. - 2016. - № 10-1. - С. 154-158.

5. Об оценке эффективности работы преподавателей в свете введения эффективного контракта / В. П. Рыжов, В. В. Терешков, Н. А. Каширина, А. А. Марьев // Высшее образование в России. 2015. - № 10. - C. 16-26.

6. Программа поэтапного совершенствования системы оплаты труда в государственных (му- ниципальных) учреждениях на 2012-2018 годы : утв. Распоряжением Правительства Российской Федерации от 26.11.2012 №2190-р. - Доступ из информ.правового портала «Гарант».

7. Рыжов, Н. Г. Анализ возможностей введения эффективного контракта для регулирования труда научных работников вузов / Н. Г. Рыжов, М. Ю. Шестопалов // Инновации. - 2013. - № 11 (181). - С. 103-109.

8. Ряписов, Н. А. Особенности реализации эффективного контракта в системе высшего образования / Н. А. Ряписов // Вестник НГПУ. - 2015. № 4 (26). - С. 18-26.

9. Сенашенко, В. С. Об эффективном контракте в высшей школе России / В. С. Сенашенко, В. Г. Халин // Высшее образование в России. 2015. - № 5. - С. 27-36.

10. Финансовые аспекты обеспечения эффективных контрактов научно-педагогических работников / А. В. Дорждеев, Н. А. Кизатова, А. Г. Лосев, В. В. Тараканов // Вестник Волгоградского государственного университета. Серия 3, Экономика. Экология. - 2015. - № 3 (32). - C. 93-103. - DOI: http:// dx.doi.org/10.15688/jvolsu3.2015.3.10.

11. Budgeting model of structural units based on normative per capita funding / S. A. Korolkov, A. G. Losev, I. M. Reshetnikova, V. V. Tarakanov // European researcher. - 2014. - № 3-1 (70). C. 498-508.

\section{REFERENCES}

1. Kizatova N.A. Dinamicheskaya model formirovaniya fonda oplaty truda professorskoprepodavatelskogo sostava vuzov [A Dynamic Model of Generating the Payroll of Faculty and Staff in Higher Educational Institutions]. Sovremennaya ekonomika: problemy i resheniya, 2016, no. 7 (79), pp. 15-24.

2. Korolkov S.A., Losev A.G., Reshetnikova I.M. Model optimalnogo planirovaniya shtata professorskoprepodavatelskogo sostava kafedr vuza [Model Optimal Planning Staff of Faculty and Staff in Higher Educational Institutions]. Vestnik Volgogradskogo gosudarstvennogo universiteta. Seriya 3: Ekonomika. Ekologiya [Science Journal of Volgograd State University. Global Economic System], 2013, no. 1, pp. 149-154.

3. Korolkov S.A., Losev A.G., Reshetnikova I.M., Tarakanov V.V. Upravlenie obrazovatelnymi programmami cherez vnedrenie uchebno-finansovykh planov [Management of Educational Programs by Means of Educational and Financial Plans]. Universitetskoe upravlenie: praktika i analiz, 2016, no. 4(104), pp. 49-55.

4. Lengardt V.A., Shemyatikhina L.Yu. Effektivnyy kontrakt $\mathrm{v}$ marketingovoy politike universiteta [Effective Contract in the Marketing Policy of the University]. Fundamentalnye issledovaniya, 2016, no 10-1, pp. 154-158. 
5. Ryzhov V.P., Tereshkov V.V., Kashirina N.A., Maryev A.A. Ob otsenke effektivnosti raboty prepodavateley $\mathrm{v}$ svete vvedeniya effektivnogo kontrakta [On Assessing Teachers' Work in the Conditions of the Effective Contract Introduction]. Vysshee obrazovanie v Rossii, 2015, no. 10, pp. 16-26.

6. Programma poetapnogo sovershenstvovaniya sistemy oplaty truda $v$ gosudarstvennykh (munitsipalnykh) uchrezhdeniyakh na 2012-2018 gody: utv. Rasporyazheniem Pravitelstva Rossiyskoy Federatsii ot 26.11.2012 №2190-r [Program of Gradual Improvement of the System of Labour Remuneration in State (Municipal) Institutions for 2012-2018: Approved by the Decree of the Government of the Russian Federation of November 26, 2012 no. 2190-p]. URL: http:/ /www. garant.ru/products/ipo/prime/doc/70169234/. (accessed May 14, 2017).

7. Ryzhov N.G., Shestopalov M.Yu. Analiz vozmozhnostey vvedeniya effektivnogo kontrakta dlya regulirovaniya truda nauchnykh rabotnikov vuzov [Analysis of Possible Introduction of an Effective Contract for Regulating the Labour of Academic Staff in Universities]. Innovatsii, 2013, no. 11 (181), pp. 103-109.
8. Ryapisov N.A. Osobennosti realizatsii effektivnogo kontrakta $\mathrm{v}$ sisteme vysshego obrazovaniya [Features of Implementing an Effective Contract in the Higher Education System]. Vestnik NGPU, 2015, no. 4(26), pp. 18-26.

9. Senashenko V.S., Khalin V.G. Ob effektivnom kontrakte v vysshey shkole Rossii [On the Effective Contract in the Higher School of Russia]. Vysshee obrazovanie v Rossii, 2015, no. 5, pp. 27-36.

10. Dorzhdeev A.V., Kizatova N.A., Losev A.G., Tarakanov V.V. Finansovye aspekty obespecheniya effektivnykh kontraktov nauchno-pedagogicheskikh rabotnikov [Financial Aspects of Ensuring Effective Contracts for Academic and Teaching Staff]. Vestnik Volgogradskogo gosudarstvennogo universiteta. Seriya 3: Ekonomika. Ekologiya [Science Journal of Volgograd State University. Global Economic System], 2015, no. 3 (32), pp. 93-103. DOI: http://dx.doi.org/ 10.15688/jvolsu3.2015.3.10.

11. Korolkov S.A., Losev A.G., Reshetnikova I.M., Tarakanov V.V. Budgeting model of structural units based on normative per capita funding. European researcher, 2014, no. 3-1 (70), pp. 498-508.

\section{Information about the Authors}

Nadezhda A. Kizatova, Candidate for a Degree, Department of Corporate Finance and Banking, Volgograd State University, Prosp. Universitetsky, 100, 400062 Volgograd, Russian Federation, kizatovanadya@yandex.ru.

Nataliya V. Loseva, Candidate of Sciences (Physics and Mathematics), Associate Professor, Department of Applied Mathematics and Computer Science, Volzhsky Institute of Humanities - Branch of Volgograd State University, 40 let Pobedy St., 11, 404133 Volzhsky, Russian Federation, losevanat@yandex.ru.

Vasiliy V. Tarakanov, Doctor of Sciences (Economics), Professor, Rector, Volgograd State University, Prosp. Universitetsky, 100, 400062 Volgograd, Russian Federation, rector@volsu.ru.

\section{Информация об авторах}

Надежда Александровна Кизатова, соискатель кафедры корпоративных финансов и банковской деятельности, Волгоградский государственный университет, просп. Университетский, 100, 400062 г. Волгоград, Российская Федерация, kizatova-nadya@yandex.ru.

Наталия Владимировна Лосева, кандидат физико-математических наук, доцент кафедры прикладной математики и информатики, Волжский гуманитарный институт (филиал) Волгоградского государственного университета, ул. 40 лет Победы, 11, 404133 г. Волжский, Российская Федерация, loseva-nat@yandex.ru.

Василий Валерьевич Тараканов, доктор экономических наук, профессор, ректор, Волгоградский государственный университет, просп. Университетский, 100, 400062 г. Волгоград, Российская Федерация, rector@volsu.ru. 